\title{
Fibre and bowel transit times
}

\author{
BY J.S. S. GEAR, A. J. M. BRODRIBB,* ALISON WARE AND J. I. MANN† \\ Department of Community Medicine and General Practice, University of Oxford, \\ 8 Keble Road, Oxford $O X 13 Q N$
}

(Received 6 March 1980 - Accepted 1 August 1980)

\begin{abstract}
1. Bowel transit time has been investigated in vegetarians and non-vegetarians and related to dietary fibre intake and the presence of diverticular disease.

2. Vegetarians who have less diverticular disease than non-vegetarians have more rapid transit times.

3. Subjects with total dietary fibre intake of more than $30 \mathrm{~g} / \mathrm{d}$ all had transit times of less than $75 \mathrm{~h}$ whereas $38 \%$ of those eating less had transit times exceeding $75 \mathrm{~h}$ and varying up to $124 \mathrm{~h}$.

4. Individuals with diverticular disease were found to have faster transit times than those without the disease.

5. The colon may respond to a fibre-depleted diet either by becoming hyperactive and prone to diverticular disease or by becoming hypoactive leading to constipation.
\end{abstract}

The stools of individuals' eating fibre-rich foods are soft and bulky and experimentally such foods have been shown to shorten bowel transit time. The transit times of members of communities eating different amounts of fibre have also been shown to vary (Burkitt $e$ t al. 1972). Studies of the relationship between diverticular disease and transit time are conflicting (Manousos et al. 1967; Eastwood et al. 1978), but this may to some extent be explained by the limitation of the earlier techniques. We have. modified the technique described by Cummings \& Wiggins (1976) to study further the association between bowel transit time, dietary fibre intake and diverticular disease.

\section{SUBJECTS AND METHODS}

Volunteers. Forty-seven of the participants in an earlier study of the relationship between asymptomatic diverticular disease and intake of dietary fibre (Gear et al. 1979) took part in this investigation. (The earlier study included fifty-six vegetarians and 264 non-vegetarians and the study population is described in detail in the 1979 publication). The subjects in the present study were not randomly selected. Approximately equal numbers of vegetarians, who had been shown to have a high dietary fibre intake, and non-vegetarians, with a low fibre intake were asked to volunteer to ensure a wide range of fibre consumption amongst participants. Selection within the vegetarian and non-vegetarian groups was based simply on proximity of the subjects' home, or place of work, to the hospital and willingness to cooperate. Of those requested to participate in this study, $98 \%$ agreed to do so. The results presented below relate to twenty-seven non-vegetarians (fourteen males and thirteen females) and twenty vegetarians (eight males and twelve females).

Bowel transit time. For the measurement of bowel transit time, it was initially intended to use the single-stool technique described by Cummings \& Wiggins (1976). This method entails swallowing twenty radio-opaque markers of known shape at a specified time on three consecutive days (rods on day 1 , circles on day 2, cubes on day 3 ). The whole of the first stool passed after waking on the fourth day is collected in a plastic bag and the time of

- Present address: Plymouth General Hospital.

$\dagger$ For reprints. 
collection recorded. The bag and contents are X-rayed, the numbers of different-shaped markers counted and transit time $(t t)$ calculated according to the following formula:

$$
t t=\frac{\left(\mathbf{R}_{1} \times t_{1}\right)+\left(\mathbf{R}_{2} \times t_{2}\right)}{\mathbf{R}_{1}+\mathbf{R}_{2}},
$$

where $R_{1}, R_{2}$ are the numbers of the two markers present in the stool in the largest numbers, $t_{1}$ is the time from ingestion of the $R_{1}$ markers to collection of stool and $t_{2}$ is the time from ingestion of the $\mathbf{R}_{2}$ marker to collection of stool.

After some of the subjects had been investigated using the three-marker technique, it became evident that an appreciable proportion had transit times of $70-90 \mathrm{~h}$, suggesting that some of them might have been shown to have longer transit times had a more sensitive method been used. We therefore repeated the studies in these subjects using five different markers instead of three. The additional markers were taken on days four and five and the stool collected the following day. Fig. 1 compares the bowel transit time using the three-marker and five-marker techniques; the three-marker transit time was estimated from the same stool specimen by reference to the latter three markers only. Short transit times were usually not affected by the introduction of the additional markers, but this technique revealed a far greater spread of times for those individuals with longer transit times.

Dietary information. Dietary information was collected using a modification of Burke's dietary history (Burke, 1974). A comprehensive questionnaire (available from the authors) concerned with recent food consumption was completed during a semi-structured interview, divided into three parts. The first part consisted of the recall of a typical week's diet; the week being divided into working and non-working days with each day being divided into seven periods: (1) on waking, (2) breakfast, (3) breakfast to midday meal including drinks and snacks, (4) midday meal, (5) midday meal to evening meal including drinks and snacks, (6) evening meal, (7) evening meal and bed-time including drinks and snacks. The interviewer did not ask leading questions during this part of the interview. A typical question was: What do you have for breakfast on working days? Both qualitative and quantitative information was elicited, visual aids being used to facilitate the estimation of portion sizes. After all spontaneous information had been noted, a check list, prepared for the meal in question was offered. Particular care was taken to identify the types of bread, flour and breakfast cereals being eaten.

The second part of the interview consisted of a more formal cross-check of foods of particular interest which might have been forgotten, possibly because of seasonal variation. The final part of the interview consisted of questions about stability of diet, changes in major foods over time and life-style (e.g. the number of meals eaten away from home each week). Each interview lasted approximately $1 \mathrm{~h}$.

This method of dietary assessment was validated against a 3-week dietary diary in a number of volunteers who recorded all food and drink in a pocket-size notebook. The quantities of each item were noted in either household measures or purchase weights and were written down at the time of eating to avoid the need for recall.

Proximate food constituents and fibre content were calculated from standard values obtained from the McCance and Widdowson tables (Paul \& Southgate, 1978). The correlation coefficient between total dietary fibre consumption assessed by means of the 3-week dietary diary and the comprehensive questionnaire was $\mathbf{0 . 8 3}$.

Radiological technique. The presence of diverticular disease was determined by a barium-follow-through technique (Ardran et al. 1978). The presence of one or more diverticula in the distal colon was regarded as evidence of the disease.

Statistical analysis. A non-parametric method (the Wilcoxon-rank-sum-test) was used when testing statistical significance. 


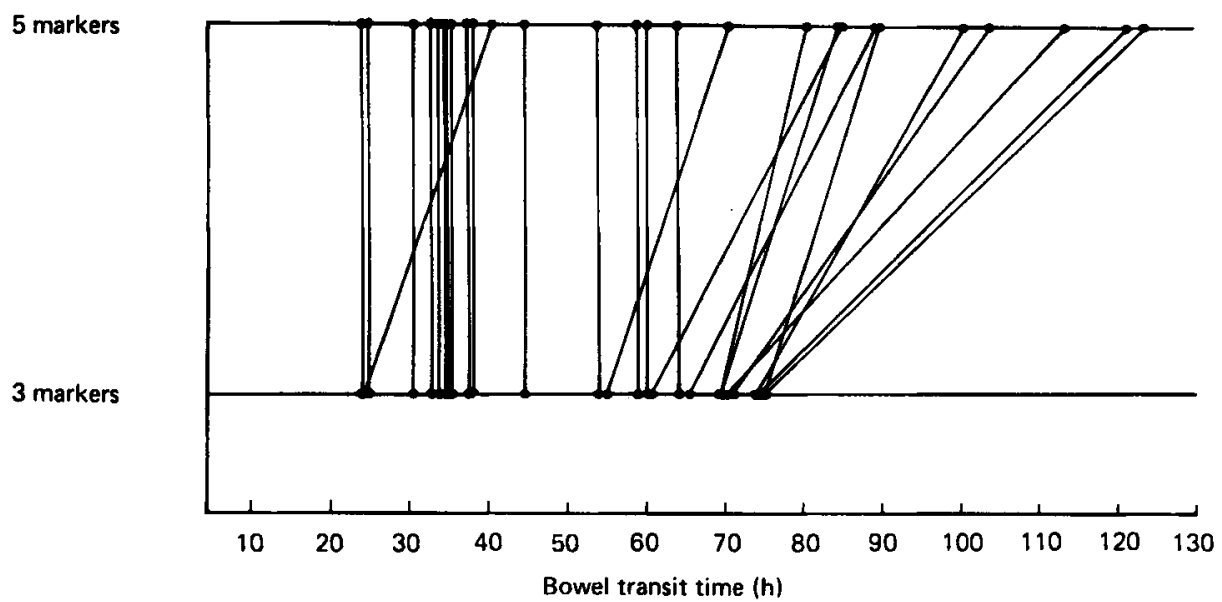

Fig. 1. Bowel transit time (h) measured by three-marker and five-marker techniques (for details, see p. 77) in twenty-seven individuals.

(a)
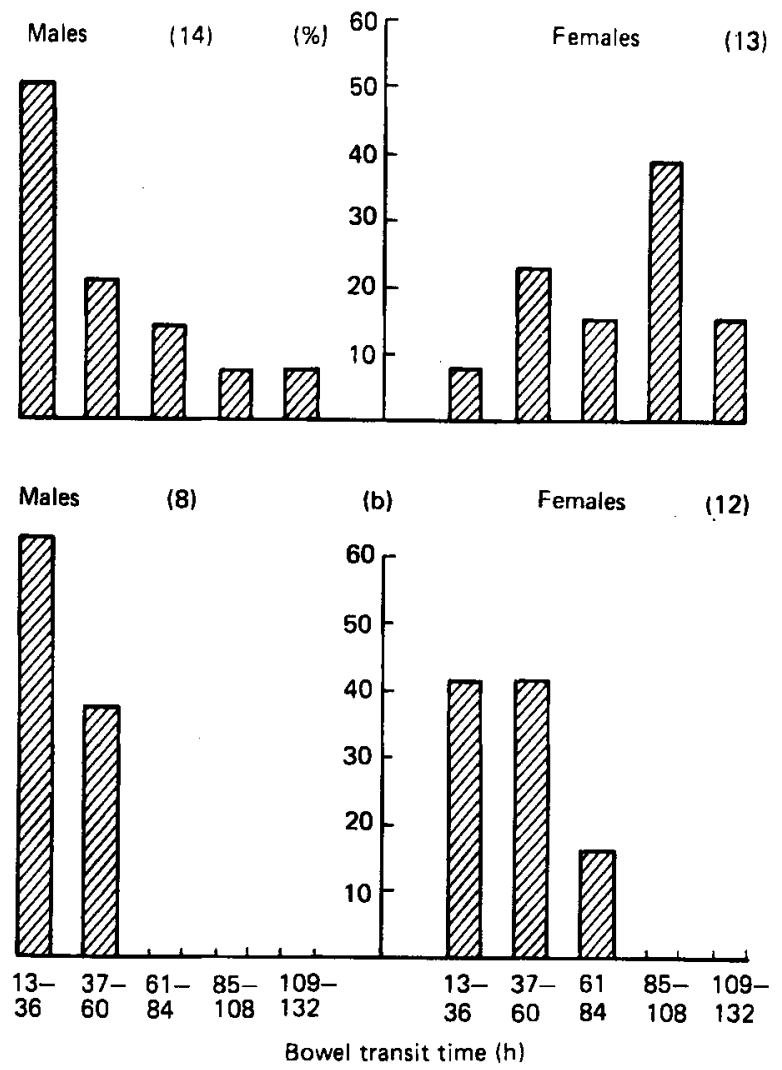

Fig. 2. Comparison of bowel transit times (h) in male and female non-vegetarians (a) and vegetarians (b). Values in parentheses are nos. of subjects participating. 

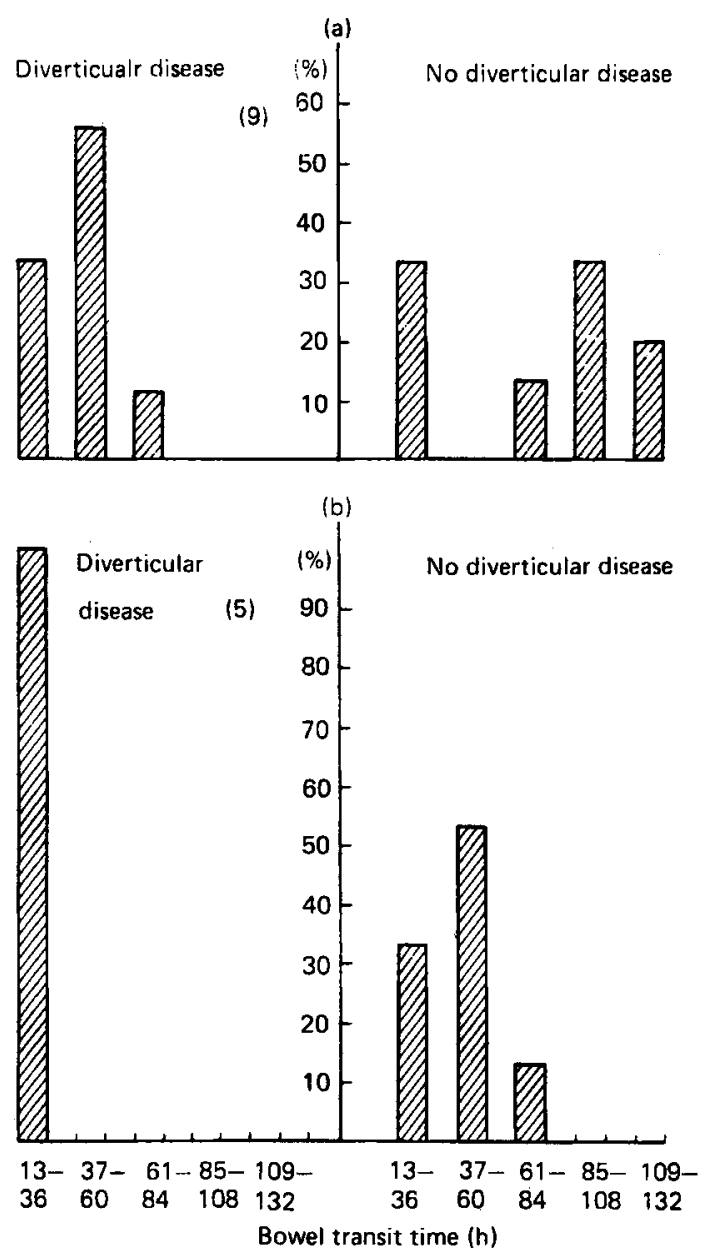

(15)

Fig. 3. Comparison of bowel transit times (h) in individuals with and without distal diverticular disease; non-vegetarians (a), vegetarians (b).

\section{RESULTS}

The distribution of bowel transit times (mean \pm SD) for the non-vegetarians was $63.8 \pm 32 \cdot 1 \mathrm{~h}$ and for the vegetarians $40.6 \pm 13.4 \mathrm{~h}$. The difference between the two groups was significant at $P<0.02$. Males tended to have faster transit times than females (Fig. $2)$ and for the non-vegetarians this was statistically significant $(P<0.02)$. There was no association between age and transit time. Fig. 3 shows that subjects with diverticular disease had faster transit times than those without the disease. Among the vegetarians this difference was statistically significant $(P<0.002)$. The small numbers of observations precluded a reliable analysis of the results for the sexes separately.

The relationship between transit time and the intake of dietary fibre is shown in Fig. 4. Individuals eating less than $30 \mathrm{~g}$ fibre daily showed a wide range of transit times whereas those eating larger amounts had a narrower range, with none exceeding $75 \mathrm{~h}$. The values were too few to permit an examination of the correlation between the different constituents of dietary fibre and transit times. Fat, protein and carbohydrate intake and total energy consumption did not appear to affect bowel transit time significantly. 


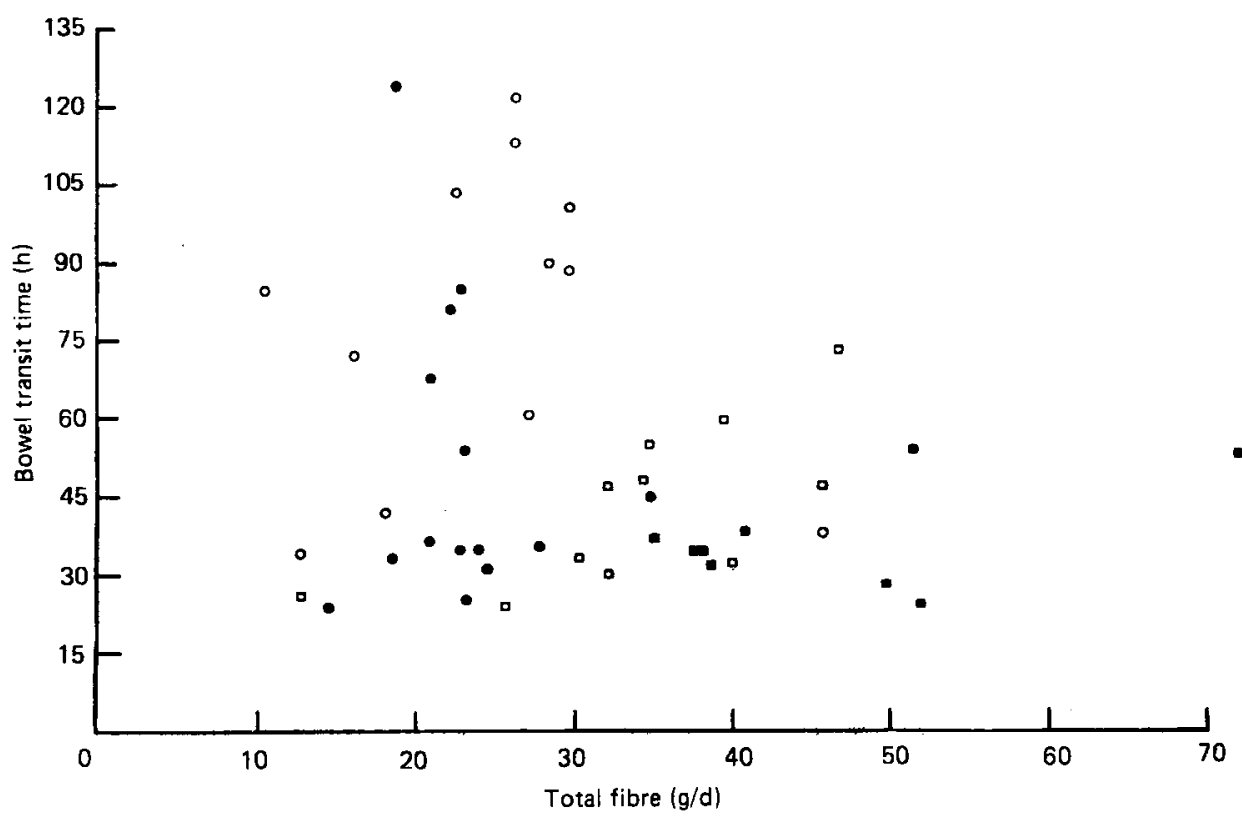

Fig. 4. Bowel transit time (h) $v$, total fibre consumption (g/d) in forty-five individuals. $O$, non-vegetarian female; $O$, non-vegetarian male; $\square$, vegetarian female; $\square$, vegetarian male.

\section{DISCUSSION}

The first interesting finding in this study relates to the methodology. The five-marker technique appears to be more satisfactory than the three-marker technique for estimating bowel transit times in communities where a large proportion of individuals have slow transit times. This observation may be relevant to other studies in which important conclusions have been based on results obtained using the original three-marker methodology. For example, findings have recently been reported for two groups of Scandinavians, one with a high fibre intake at low risk of colon cancer and another with a low fibre intake at appreciably higher risk (International Agency for Research on Cancer, 1977). Transit times were similar in the two groups and the authors concluded that transit time was probably not relevant in the aetiology of colon cancer. The authors also observed a bimodal distribution of transit times. Because the three-marker method was used, it is conceivable that both represent artifacts of technique. More work is clearly required to confirm or refute this suggestion.

The present study confirms that a high intake of dietary fibre is associated with more rapid transit times. Vegetarians (amongst whom we had previously demonstrated a high fibre intake, Gear et al. 1979) had more rapid transit times than non-vegetarians. Furthermore when considering all forty-seven subjects (regardless of whether or not they were vegetarians) those with a total fibre intake of more than $30 \mathrm{~g} / \mathrm{d}$ had transit times within a narrow range with an upper limit of $75 \mathrm{~h}$. Both vegetarians and non-vegetarians with diverticular disease had significantly faster transit times than those without the disease. These findings appear to conflict with our earlier results which suggested that a high intake of dietary fibre is protective against diverticular disease.

A possible explanation is suggested by the results in Fig. 4 ; i.e. that individuals may adapt to fibre-depleted diets in one of two ways: the bowel may either become hyperactive producing rapid transit times, or unresponsive producing slow transit times. The hyperactive 
bowel would result in a colon of narrow diameter with a high intraluminal pressure. This condition would predispose to the formulation of diverticula. The hypoactive bowel would result in constipation, with the infrequent passage of hard motions.

This explanation is compatable with the dietary hypothesis in relation to carcinoma of the colon (Burkitt, 1974). Undoubtedly colon cancer has a multifactorial aetiology and a slow transit time might predispose to the production of colonic carcinoma. If this was the situation, diverticular disease might be expected to be less common in individuals with colon cancer than in the rest of the community. The subjects in this study have been used as a control group for comparison with colon cancer patients and have in fact been found to have more diverticular disease than the cancer cases (Latto, personal communication).

Sex differences in bowel transit time have not been reported previously and there is no obvious explanation for females having slower transit times than males. It is possible that chance could explain the difference since the numbers studied were relatively small. However, the findings are consistent with an earlier observation (Gear et al. 1979) that the association between diverticular disease and fibre-depleted diets was particularly strong in females. Furthermore, the findings appear separately in both vegetarians and non-vegetarians. It is common clinical observation that women are especially prone to complaints of constipation.

The authors are most grateful to Professor Martin Vessey, Dr John Cummings and Mary Tudor Hart for all their help and advice and to the Vegetarian Society of the United Kingdom and many other volunteers who participated in the study. The stools were X-rayed in the Department of Radiology, University of Oxford, with the kind help of Dr Gordon Ardran and Pel Fursdon.

\section{REFERENCES}

Ardran, G. M., Nolan, D. J., Gear, J. S. S., Fursdon, P. S. \& Brodribb, A. J. M. (1978). Br. J. Radiol. 51, 472. Burke, B. S. (1974). Cancer 28, 3.

Burkitt, D. P., Walker, A. R. P. \& Painter, N. S. (1972). Lancet ii, 1408.

Cummings, J. H. \& Wiggins, H. S. (1976). Gut 17, 219.

Eastwood, M. A., Smith, A. N., Brydon, W. G. \& Pritchard, J. (1978). Lancet i, 1181.

Gear, J. S. S., Ware, A., Fursdon, P., Mann, J. I., Nolan, D. J., Broadribb, A. J. M. \& Vessey, M. P. (1979). Lancet i, 511 .

International Agency for Research on Cancer. (1977). Lancet ii, 207.

Manousos, O. N., Truelove, S. C. \& Lunsden, K. (1967). Br. med. J. ii, 760.

Paul, A. A. \& Southgate, D. A. T. (1978). McCance and Widdowson's The Composition of Foods. London: H.M. Stationery Office. 